\title{
Wissenschaftliche Revolution und gesellschaftlicher Wandel
}

\section{Kommentar zu Gerhard Harig: Die beiden Aspekte der wissenschaftlichen Revolution des 17. Jahrhunderts und die Gegenwart (1966)}

Wolfgang Krohn

Der Aufsatz von Gerhard Harig bietet eine erstaunlich frühe Auseinandersetzung mit Thomas S. Kuhns The Structure of Scientific Revolutions. 1962 war das Werk erschienen, ohne zunächst auf große Resonanz zu stoßen. Erst 1967 brachte Suhrkamp die deutsche Übersetzung heraus. Etwa zur gleichen Zeit setzte der beispiellose internationale Erfolg ein, den man vermutlich mit den revolutionären Attitüden der 1968er-Bewegung in Verbindung bringen kann, auch wenn weder der Inhalt noch die Einstellungen des Autors in unmittelbarer Nähe zu den Themen und Zielen dieser Zeit stehen. Da Harig bereits 1966 starb, konnte er die steile Karriere des Kuhn'schen Buches nicht mehr erleben und interpretieren. 1965 war sein Ausgangspunkt für die Auseinandersetzung mit Kuhn die ,wissenschaftlich-technische Revolution der Gegenwart”. Verwundert wird man heute fragen: Welche war denn das? Wie sich aus der Gesamtanlage seines Beitrags ergibt, war mit dieser Benennung kein markanter

Der Nachdruck des Originalbeitrags ist in diesem Heft dem Kommentar vorangestellt und online unter dem DOI:10.1007/s00048-010-0019-0 zu finden. 
innerwissenschaftlicher Umbruch gemeint, sondern der mit dem Aufbau des Sozialismus einhergehende Umbau von Wissenschaft und Technik. Und etwas genauer: 1963 begab sich die DDR mit dem „Neuen ökonomischen System der Planung und Leitung” (NÖSPL) in eine Erneuerungsphase der Wirtschaftsentwicklung, die die inzwischen deutlich erkennbaren Lähmungserscheinungen des zentralistischen und dirigistischen Modells auflösen sollten. Es kam nicht nur zur Anwendung und Anerkennung einzelner marktwirtschaftlicher Anreize und struktureller Regulierungsmechanismen, sondern auch zu einer „experimentell” genannten Implementierungsstrategie des neuen Modells in ausgesuchten Betrieben. Es war wohl das erste und einzige? - Mal, dass sich die DDR-Führung entschloss, Reformschritte nicht zu deduzieren, sondern auszuprobieren. ${ }^{1}$

Zur selben Zeit (1961) erschien in der DDR die deutsche Übersetzung von John D. Bernals Werk Science in History dessen Botschaft für die Gegenwart Fortschritt durch Wissenschaftsplanung war. Wie ernsthaft die Idee verfolgt wurde, für den Aufbau des Sozialismus auf die Planung wissenschaftlichen Fortschritts $\mathrm{zu}$ setzen, zeigen auch die Schrift Sozialismus-Wissenschaft-Produktivkraft, die 1963 von einem wissenschaftlichen Kollektiv am Institut für Gesellschaftswissenschaften beim Zentralkomitee der SED verfasst wurde, und der parallel dazu entstandene Tagungsbericht Die Entwicklung der Wissenschaft zur unmittelbaren Produktivkraft auf die Harig in Fußnote 20 hinweist. Wissenschaftsförderung auf wissenschaftlicher Grundlage („Wissenschaftswissenschaft”, science of science, wurde diese ambitionierte Selbstüberschätzung in Ost wie West genannt) und Wirtschaftsdynamik mit experimenteller Lernbereitschaft verkoppelt im welthistorischen Projekt des Sozialismus das ist die im ersten Satz von Harig angesprochene ,wissenschaftlichtechnische Revolution der Gegenwart".

In diesem Kontext ist Harigs Auseinandersetzung mit Kuhn nicht rein akademischer Natur. Harig möchte eher überprüfen, wie weit diese Position aus der westlichen Welt mit ihrem markanten und provozierenden Titel zusätzliche Unterstützung bot. Danach ging es Harig auch weniger um die von Kuhn thematisierten Paradigmenwechsel im Verlauf der neuzeitlichen Wissenschaft als vielmehr um die so genannte wissenschaftliche Revolution im 17. Jahrhundert, die mit den Namen Galilei, Bacon und Descartes verbunden ist. Die Struktur dieses säkularen Umbruchs und ihre Ähnlichkeit mit der des stattfindenden Wandels in der beginnenden sozialistischen Gegenwart, ist das Kernthema des Aufsatzes.

Ich beginne dennoch mit einer kurzen Rekonstruktion von Harigs Sicht auf Kuhn. Da fällt zunächst auf, dass die textliche 
Paraphrase des Schlüsselzitats in der Anmerkung 2 ausspart, dass nach Kuhn konkurrierende Paradigmen inkompatibel sind. „Über den Ausgang der Kämpfe entscheidet letzten Endes der Vergleich beider Konzeptionen mit der Erfahrung und mit den Tatsachen" (S. 3). Damit wird - eher nebenbei - dem Kuhn'schen Konzept der Giftzahn gezogen. Denn für Kuhn war der zentrale Punkt, dass es keinen dritten Standpunkt gibt, von dem aus man neutral die Vor- und Nachteile der konkurrierenden Paradigmata abwägen kann. Ob etwas von Vorteil ist (beispielsweise für den experimentellen Beleg eines Geltungsanspruchs), lässt sich nach Kuhn nur im Kontext eines Paradigmas bewerten. „Wenn Paradigmata in eine Diskussion über die Wahl von Paradigmata eingehen - und sie müssen es ja - dann ist ihre Rolle notwendigerweise zirkulär" (Kuhn [1962] 1967: 106). Kuhn hat mit dieser Zirkularität nicht behaupten wollen, dass Paradigmata keinen gemeinsamen Boden haben, sondern nur, dass es einen inkommensurablen Rest gibt, zu dessen Auflösung ein rationales Entscheidungsverfahren nicht hinreicht. Letztendlich sind Paradigmata nicht wahr oder falsch, sondern bestimmte Weltsichten, zu denen man sich bekennt oder nicht. Ohne diesen Giftzahn werden wissenschaftliche Revolutionen zu Phasen beschleunigter Entwicklung, bei denen Quantität in Qualität umschlagen kann, so Harig (S. 1), oder Extensität der Forschung durch Intensität ersetzt wird (so Harig auf S. 4 im Anschluss an Mikulinski). Mit dem Giftzahn weicht Kuhns Modell radikal von den Ansichten ab, nach denen sich zumindest letztendlich der kumulative Fortschritt durchsetzt - wenn auch verbunden mit „Umformung und Umbildung” (ebd.: 3). So wird bei Harig die Inkompatibilität unterlaufen durch eine Sicht der „dialektischen” Entwicklung (ebd.: Fn. 5), in der das Negierte aufgehoben wird. Mit dieser Glättung gelingt es Harig dann sogar, Kuhn als einen Realisten der „objektiven Außenwelt” (ebd.: 3) darzustellen, dessen Modell mit „den positivistischen Theorien der Verifikation oder der Falsifikation nach K. P. Popper nicht zu vereinbaren ist." (Ebd., Hervorhebung im Original.) Dies klingt nach einer visionären Vorwegnahme der großen Auseinandersetzung, die unter dem Titel Criticism and the Growth of Knowledge 1970 veröffentlicht wurde, ist aber doch nur ein Missverständnis. Denn gerade der Falsifikationismus hat als Bedingung den Vergleich mit einer theorieunabhängien Realität, während Kuhn die paradigmenübergreifende Wirksamkeit eines solchen Tests gerade bestreitet. Hier wirkt wohl das Hegel'sche Erbe des Marxismus, demzufolge in der Weltgeschichte nichts vergeblich gewesen ist, sondern in dialektischen Widersprüchen zur Gesamtentwicklung beiträgt. 
Die wichtigste Differenz zum Ansatz Kuhns ist allerdings tiefer. Während dieser die Beziehungen zwischen politischen und wissenschaftlichen Revolutionen in erster Linie als eine begriffliche Analogie konstruiert, sieht Harig konsequent Wissenschaft als Teil der Gesellschaft. Harig gewinnt diese Position an dem Paradigmenwechsel des 17. Jahrhunderts, also dem Urbild und Namensgeber der wissenschaftlichen Revolution als ein generischer Typus. Dabei ging es nach Harig ebenso stark um die Herausbildung neuer „Vorstellungen, Begriffe, Theorien und Methoden [...] wie die Entdeckung der gesellschaftlichen Funktion der Wissenschaft" (ebd.: 8). Der Begriff Entdeckung ist in diesem Zusammenhang etwas unglücklich gewählt, da man ja schwerlich die gesellschaftlichen Funktionen der vorneuzeitlichen Wissenschaften mit Blick auf die Entwicklung freier Städte seit dem Spätmittelalter, auf die höfische Kultur der Renaissance und ohnehin auf die Dienste gegenüber der Religion bestreiten kann. Es wäre also richtiger, hier von einem Funktionswechsel, als von einer Entdeckung zu sprechen. Später sieht Harig das Neue in einer „Veränderung oder genauer gesagt in einer Vertiefung und Ausweitung der gesellschaftlichen Funktionen" (ebd.: 9), was ich keineswegs „genauer gesagt” finde. Für seine Schlussfolgerung ist diese Präferenz von Kontinuität gegenüber Bruch nicht entscheidend: „Nur wenn sich das Paradigma und die gesellschaftliche Funktion der Wissenschaft gleichzeitig ändern, kommt es zu einer wissenschaftlichen Revolution." (Ebd.). Diese These gewinnt man nach Harig, wenn man die ",historische Erfahrung der großen wissenschaftlichen Revolution des 17. Jahrhunderts verallgemeinert" (Ebd.).

Nimmt man die These als eine empirische Verallgemeinerung, ist sie fragwürdig, weil sie keineswegs auf alle Beispiele passt, die teils in ihren Selbstbeschreibungen, teils in ihren historiographischen Rekonstruktionen als wissenschaftliche Revolutionen gelten. Man hätte beispielsweise Schwierigkeiten bei Darwin und bei Einstein. Nimmt man die These jedoch als Vorschlag einer begrifflichen Eingrenzung, hat sie den großen Vorteil, der Auflösung des Phänomens in beliebig viele Mini-Revolutionen entgegenzuwirken. Dieser Einwand, dass wissenschaftliche Entwicklung eigentlich eine Verkettung vieler kleiner Revolutionen und also insgesamt eine permanente Revolution sei, wurde bereits 1963 erhoben (ebd.: 9, Fn. 18) und später durch mehrere Autoren, besonders Stephen Toulmin ausgebaut. Harigs These führt gegenüber diesem Einwand also zu einer durchaus sinnvollen Eingrenzung auf historische Konstellationen, in denen radikaler gesellschaftlicher und wissenschaftlicher Wandel koinzidieren und einander bestärken. Der Preis mag für einen an Kuhn orientiertem Wissenschaftshistoriker zu hoch 
sein, weil aus der Anzahl anerkannter wissenschaftlicher Revolutionen, die etwa I. Bernard Cohen (1985) zusammengestellt hat, einige herausfallen. Aber für eine an Gesellschaftstheorie orientierte Wissenschaftsgeschichtsschreibung mag die Eingrenzung durchaus sinnvoll sein.

Der Hauptteil des Aufsatzes ist - wie der Titel verspricht eine Analyse der wissenschaftlichen Revolution des 17. Jahrhunderts mit der Absicht, die benannte Koinzidenz aufzuweisen. Harig beginnt die Analyse mit einer einfachen Frage: Warum nicht Galilei, sondern Francis Bacon als Schlüsselfigur dieser Revolution in den Wahrnehmungen des späten 17. und des 18. Jahrhunderts? Wissenschaftshistorisch hätte es nahe gelegen jenen zu küren, der explizit und fundiert das "mathematisch-experimentelle” gegen das ,aristotelisch-scholastische” Weltbild stellte (ebd.: 4-5). Der moderne Wissenschaftshistoriker würde hier nicht lange zögern. Aber in den Selbstbeschreibungen der Zeit wuchs Bacons Bedeutung ständig an, bis schließlich mit der Prägung des Begriffs Baconismus in der Enzyklopädie die Aufklärung die historische Führerschaft für denjenigen festschrieb, der als erster und unzweideutig die Forderung nach einer neuen Wissenschaft aus dem Wandel der Gesellschaft ableitete und den Erfolg gesellschaftlichen Wandels von den Erfolgen der Forschung abhängig sah. Bacon hat mehrfach betont, dass es eine "Schande” wäre, wenn die „Entdeckungen der geistigen Welt” auf ihren alten Kreis beschränkt blieben, nachdem für die materielle Welt der ganze Erdkreis eröffnet wurde (Bacon 1990, Erster Teil, Aph. 84). Sowohl die materielle als auch die geistige Expansion läuten eine neue Revolution sein, wobei dieser Begriff halb „Epoche” oder „Umschwung”, halb ,neue Weltordnung” trägt.

Harig stärkt seine Interpretation Bacons mit Hinweisen auf die wegweisenden Untersuchungen von Farrington und Crowther (Fn. 14, 15). Aus Sicht der heutigen Forschung wäre vielleicht zu korrigieren, dass Bacon in seinen philosophischen Auffassungen weit weniger radikal war, als ihm die Gloriole der Aufklärung andichtete. Man hätte entgegenzuhalten, dass hinter seiner radikalen Forderung nach einer neuen gesellschaftlichen Funktion der Wissenschaft ein ambivalenter Umgang mit klassischem Wissen stand. So ist etwa seine Methode der Induktion vor allem deswegen problematisch, weil sie sich an den Regeln (leges) der Rhetorik der Renaissance, insbesondere Petrus Ramus, orientiert, statt an Beispielen induktiver Forschung. Aber gerade diese Ambivalenz macht Bacon zu dem Denker, der fast im alchemistischen Sinne eine Transformation leistet: die Übersetzung einer alten Wissensform in 
eine neue mit Mitteln, die vor allem in der alten Form selbst liegen. Für die Wissenschaft an sich ist der radikal neue Grundgedanke, das jedes Wissen aus der Forschung stammt und zu Forschung führt, also essentiell Wissen im Wandel ist.

Insgesamt gibt es auch aus heutiger Sicht keine grundlegenden Einwände, die wissenschaftliche Revolution des 17. Jahrhunderts erstens so zu bezeichnen (trotz der Tendenz einiger Wissenschaftshistoriker und Wissenschaftshistorikerinnen, alle Einschnitte mit evolutionären Interpolationen zu glätten) und zweitens mit gesellschaftlichen Veränderungen zu verknüpfen. So bleibt am Ende die Frage, ob eine Strukturähnlichkeit zwischen dieser Revolution und dem intendierten Aufbruch der 1960er Jahre in eine gesellschaftliche Entwicklung besteht, die auf Wissenschaft als primärer Produktivkraft setzt, indem einerseits Wissenschaft planmäßig organisiert und andererseits Produktion planmäßig verwissenschaftlicht wird. Wir erinnern uns, dass im Westen nicht weniger als im Osten diese Ideen einer geplanten Forschung und organisierten Modernisierung verbreitet waren. Insofern besteht eine Strukturähnlichkeit zwischen dem Baconismus des 17. Jahrhunderts und der Planungseuphorie der Zeit von ungefähr 1960 bis 1980. Aber die Vorstellungen der Planbarkeit mussten im weiteren Verlauf erheblich modifiziert werden, weil man die Effizienz der Planlosigkeit unterschätzte. Richtig ist, dass die Bedeutung der anwendungsorientierten Forschung immer weiter zugenommen hat. Aber zugleich - man kann beinahe sagen: erstaunlicherweise - hat die Bedeutung der durch Neugier getriebenen, an Zufällen und Gelegenheiten statt an Planungen orientierten Forschung nicht abgenommen. So wie Bacon sich in seinem Entwurf getäuscht hatte und man dessen gewahr wurde, indem man ihn umzusetzen suchte, so hat sich der Produktivkraft-Entwurf durch seine Umsetzung als Täuschung herausgestellt. In beiden Fällen führten beziehungsweise führen die wissenschaftlich-gesellschaftlichen Revolutionen zur Suche nach neuen institutionellen Mustern, die nicht vorhergesehen wurden. Obwohl sich für die gegenwärtige Entwicklung der Begriff der Wissensgesellschaft bereits eingespielt hat, ist die Suche nach der institutionellen Neuordnung zwischen Forschung und Innovationsdynamik keineswegs abgeschlossen.

\section{Anmerkungen}

1 Zum politischen Schicksal der ökonomischen Reformen siehe Roesler 2005. 


\section{Literatur}

Bacon, Francis, 1990. Neues Organon, lateinisch-deutsch, hg. von Wolfgang Krohn, Hamburg: Felix Meiner Verlag.

Cohen, I. Bernard 1985. Revolution in Science. Cambridge, MA.: Harvard University Press.

Kuhn, Thomas S., [1962] 1967. Die Stuktur wissenschaftlicher Revolutionen. Frankfurt a. M.: Suhrkamp Verlag.

Roesler, Jörg, 2005. Neues Denken und Handeln im Neuen Ökonomischen System (NÖS). In: Hans-Christoph Rauh und Peter Ruben, Hg., Denkversuche. DDRPhilosophie in den 60er Jahren. Berlin: Ch. Links Verlag, 51-77.

\section{Wolfgang Krohn}

Institut für Wissenschafts- und Technikforschung

Universität Bielefeld

PF 100131

33501 Bielefeld

Deutschland

E-Mail: wolfgang.krohn@uni-bielefeld.de 